\title{
Advanced Guidance and Control Project for Reusable Launch Vehicles
}

\author{
Draft \\ John M. Hanson ${ }^{1}$
}

\begin{abstract}
The goals of this project are to significantly reduce the time and cost associated with guidance and control design for reusable launch vehicles, and to increase their safety and reliability. Success will lead to reduced cycle times during vehicle design and to reduced costs associated with flying to new orbits, with new payloads, and with modified vehicles. Success will also lead to more robustness to unforeseen circumstances in flight, thereby enhancing safety and reducing risk. There are many guidance and control methods available that hold some promise for improvement in the desired areas. Investigators are developing a representative set of independent guidance and control methods for this project. These methods are being incorporated into a high-fidelity reusable launch vehicle simulation. A simulation flyoff is being conducted across a broad range of flight requirements. The guidance and control methods that perform the best will have demonstrated the desired qualities.
\end{abstract}

\section{Introduction}

Two of NASA's goals for the next 10 years are to reduce launch costs by one order of magnitude and at the same time to increase safety by two orders of magnitude. While a new generation of expendable launch vehicles will reduce launch costs, there is a limit to how much can be saved as long as the engines, avionics, etc. are thrown away with each launch. Although there is no guarantee that a given reusable launch vehicle will be any cheaper than expendables, the only way that significant reductions in cost will be possible is through flight of robust reusable launch vehicles.

A number of commercial reusable launch vehicles are currently at some level of design and are awaiting sufficient financing. Others are sure to follow as NASA's research in this area increases. This increasing emphasis on reusability, coupled with the desire to reduce turnaround times and costs for these vehicles, demands a robust guidance and control system that can adapt to changes easily.

Flight of a reusable launch vehicle is significantly more complicated than flight of an expendable launch vehicle. In addition to the vehicle ascent, numerous abort trajectories are possible for various engine failure scenarios. Some of these are constrained due to reduction in control power in ways the nominal trajectory was not. Entry from various orbits to potentially multiple landing sites must be planned for, with thermal and structural constraints observed. If there is to be a two orders of magnitude improvement in safety, then the vehicle is likely to have increased control power for reconfiguring due to failure of at least some control effectors during certain phases of flight. The guidance and control must be able to reconfigure for these failures and land the vehicle safely. For increased safety, the guidance and control must also be able to adapt to unforeseen

\footnotetext{
' Aerospace Engineer, NASA Marshall Space Flight Center/TD54, Huntsville, AL 35812, member AIAA.
} 
circumstances during flight, such as poorly modeled vehicle systems or unexpected vehicle modes.

Classical control methods have been used for launch vehicles in the past, including the world's only reusable launch vehicle, the Space Shuttle. Use of classical methods requires intense analysis whenever there are any changes. During a reusable launch vehicle design, any change in vehicle mass properties, aerodynamics, engine performance, or trajectory requires a prolonged design cycle where stability analysis is performed, gains are derived, and simulations are performed. The nominal ascent and entry are a small part of the problem. Abort trajectories must be analyzed separately, and there are a multitude of these. The vehicle flies a bit differently for each abort case. A large number of modern control design methods exist, all of which claim some advantage to classical methods in terms of being more robust and more adaptable to changes.

On the guidance side, ascent of launch vehicles is typically handled by an openloop and then a closed-loop, optimal flight phase. These methods work fine for expendable launch vehicles and yield excellent performance. However, they are not suited to abort trajectories in the sense that early aborts require separately designed trajectories (assuming a vehicle that has the physical capability to withstand loss of an engine). These separate trajectories may have to meet different constraints due to loss of control power with an engine out. The effort expended to work abort trajectories (in order to derive the appropriate open-loop profiles) can be significant. In addition, the closed-loop guidance may or may not meet the needs of all aborts (such as return-to-launch-site or abort downrange).

Entry guidance on a reusable launch vehicle has been demonstrated on the Space Shuttle. The Shuttle procedure works well in terms of handling entry dispersions and successfully flying the vehicle to the Terminal Area Energy Management (TAEM) interface. However, research has been conducted more recently that shows several possibilities for entry guidance that the Shuttle guidance does not consider. Targeting for the TAEM interface can be done in a more robust fashion, so that a vehicle with aerodynamic properties that are not as good as those of the Shuttle can still successfully land. Entry guidance can do more to consider thermal constraints so that different thermal protection systems become viable. The entry flight path can be shaped to avoid certain populated areas on the ground.

In contrast to the entry phase, similar improvements for the TAEM phase guidance have not been identified, at least to the author's knowledge. The Shuttle TAEM phase guidance is very robust to dispersions (high and low energy cases), and thermal considerations are no longer an issue.

The $X-33$ reusable launch vehicle technology demonstrator is meant to demonstrate a number of technologies for future reusable launch vehicles. Among these are aerospike main engines, a lifting body shape, metallic thermal 
protection, composite structure, and automated flight operations. The fast pace of the program did not allow for investigating the different guidance and control possibilities and choosing the best. Methods were used that could be implemented quickly in simulation with high confidence that they would work. This led to an early working simulation that could be used for vehicle design decisions. The control design [Ref. 1] and the ascent guidance design [Ref. 2] use standard (classical) methods. The entry guidance [Ref. 2] is similar to that used on the Shuttle, with some improvements. In addition to the open-loop ascent, a function in the X-33 Mission Manager computer simulates the rest of the flight and determines whether the ascent trajectory needs to be reshaped [Ref. 2]. This function automatically reshapes the ascent if necessary. It does not, however, take into account differing trajectory design constraints that may be necessary in the event of an early engine-out (powerpack-out) abort.

The Advanced Guidance and Control (AG\&C) Project is intended to examine as many of the potential alternate methods as possible (within financial constraints) and to determine which of these supply the desired improvements that will make reusable launch vehicle design and flight cheaper and more reliable. Company proprietary methods are not being considered, as the desire is to make the best methods available for any reusable launch vehicle developer. The test series for the various methods is designed to give confidence that these schemes can be confidently chosen for use on a new vehicle.

The AG\&C Project is a $1 \frac{1}{2}$-year effort that began in April 1999 and is scheduled for completion in September 2000. The goal is to determine, through simulation fly-off, which advanced methods will reduce cost and increase safety (reduce risk) for future reusable launch vehicles.

The demonstration of the cost savings will be through demonstration that the design ${ }^{2}$ is able to fly multiple vehicle types, multiple mission scenarios, dispersions, and aborts in a robust fashion. That is, significant ground analysis is not required to fly different missions with dispersions and aborts, and the design works for multiple vehicle types. Preflight work will always be required: as a minimum, automated planning algorithms and verification simulations. The objective here is to remove development, tuning, and analysis of the algorithms prior to flying different missions. We should know that the algorithms will be able to handle the different missions and require simply development of I-loads ${ }^{3}$ and verification in processes that can be automated.

The demonstration of safety enhancements will be through demonstration that the design leads to reduction in flight risk and increase in robustness. The ability

\footnotetext{
${ }^{2}$ The term "design", as used here, refers to a general class of guidance or controller and corresponding support software, e.g., H-infinity, symbolic inversion, or sliding mode control, with the I-loads left as unspecified until a trajectory/vehicle is specified.

${ }^{3}$ I-loads are "initialization loads," equivalent to the input to a computer program but for the flight software. These are the items that can change from flight to flight and are not part of the verified flight software itself.
} 
to successfully handle large dispersions, abort situations, and failures will be part of this. The winning designs will increase the scope of scenarios that can be accommodated. Further safety enhancements will be demonstrated by tests that show these algorithms can adapt to unforeseen problems with vehicle modeling pre-flight and with unforeseen flight conditions that can physically be accommodated but would cause loss of vehicle with previous guidance and control designs.

\section{Candidate Algorithms}

The algorithms being implemented are as follows:

Ascent Guidance

The following guidance algorithms could potentially fly all ascent trajectories, or alternatively could fly abort cases and be combined with an existing optimal vacuum guidance for orbital injection.

Hybrid guidance. Uses optimal control, combined with collocation techniques, to optimize the ascent/abort trajectory. Primary investigator is Mr. Greg Dukeman/NASA MSFC, who is performing this effort as part of the requirements for his doctorate at Georgia Institute of Technology, under the direction of Professor Anthony Calise. It has the potential of being able to determine modified profiles, with modified targeting, closed-loop on-board the vehicle.

Discrete Parameter Neighboring Optimal Control (Ref. 3). This method uses optimal control to steer to the optimal trajectory. It is being worked under a separate program, but is being applied to this effort. Dr. Dan Moerder at NASA Langley is the principal investigator.

Trajectory Following Guidance. This method follows a design trajectory, allowing any input trajectory to be followed. It extends work done by Lu (Ref. 4) to three dimensions. The guidance law is based on a continuous-time predictive control concept. Mr. Dan Coughlin at NASA MSFC is the principal investigator.

\section{Entry Guidance}

Predictor-Corrector. This procedure chooses the control variables to target desired end conditions and takes intermediate constraints into account. It performs a numerical integration to determine the effect of its commands. It should yield reduced maneuvering with good targeting of end conditions and robustness to dispersions. This method has been worked extensively in the literature (Refs. 5-9). The primary investigator is Mr. Curtis Zimmerman/NASA MSFC. 
Entry Guidance by Trajectory Regulation (Ref. 10). This method tightly regulates the actual entry trajectory about a reference trajectory. Control along the trajectory is based on receding horizon control. The principal investigator is Professor Ping Lu of lowa State University.

Reduced-order Predictive/Tracking Entry Guidance (Ref. 11). This is an extension of the Shuttle guidance. It improves tracking by integrating both angle of attack and bank angle as primary controls and by eliminating discontinuous bank reversals. It allows design of the ground track to avoid populated areas. Professor Ken Mease of the University of California at Irvine is the principal investigator.

\section{Control}

Sliding Mode Controller (Ref. 12). This control is based on sliding mode theory. It is nonlinear and uses a Lyapunov design technique. A dual-loop architecture is used, with a slow attitude loop and a fast rate loop. Professor Yuri Shtessel of the University of Alabama in Huntsville is the principal investigator.

Fault Tolerant Nonlinear Adaptive Controller (Ref. 13). This controller uses a combination of feedback linearization and neural networks. No gain scheduling is required. Two control architectures are to be investigated: model inversion and parameter modification to the baseline control law. This method was developed originally under the Air Force RESTORE program. The principal investigator is Professor Anthony Calise of the Georgia Institute of Technology.

Dynamic Inversion (Ref. 14). This method uses dynamic inversion in an explicit model-following framework. It uses a direct adaptive neural network to regulate inversion error. Control derivatives are identified real-time. It uses null-space injection control allocation. This method was also developed originally under the Air Force RESTORE program. The principal investigator is Dr. David Doman of the Air Force Research Lab at Wright Patterson AFB. This effort is being funded independently but is being applied to the Advanced Guidance and Control Project.

Gain-Scheduled Linear Time Invariant (Ref. 15). This controller is based on robust $\mathrm{LTl}$ theory where control allocation is via an optimized gain schedule. An early benefit of this work was quick feedback to trajectory designers as to the angles of attack that are controllable for entry trajectory design. The principal investigator is Professor A. Scottedward Hodel of Auburn University.

Linear Parametrically Varying Controller (Ref. 16). This controller is a continuous gain-scheduled controller. Gains are a function of Mach and dynamic pressure. This method is being worked by Mr. Asif Ahmed of the Jet Propulsion Laboratory and Professor Roy Smith of UC Santa Barbara. 
Controller Design by Trajectory Linearization (Ref. 17). This method uses nonlinear tracking and decoupling control by trajectory linearization. It can be viewed as the ideal gain-scheduling controller designed at every point on the flight trajectory. It uses a singular perturbational approach. The principal investigator is Professor Jim Zhu of Louisiana State University.

The above algorithms were chosen for several reasons. In all cases except for one, work on those algorithms, as applied to reusable launch vehicles, was already being done, was paid for outside this effort, or leveraged off of significant past investment. The one other design was part of a previous effort to do control research for reusable launch vehicles and was carried along. The collection represents an attempt to gather the most promising techniques that are far enough along that they can be implemented quickly for a realistic space vehicle. Sufficient time and money was not available to openly compete for other methods than the ones chosen, and it is doubtful that others would be as far along relative to the total $1 \frac{1}{2}$ year duration of the effort.

\section{Algorithm Evaluation}

Many of the criteria for a successful flight can be tested automatically. Discipline engineers will need to review actual flight data to determine success on other parameters. Not all parameters will be actively constraining for all phases of flight. For example, most of the thermal indicators are important during entry only. For some of the alternate vehicles, X-33 indicators will be used and the values will be compared to use of nominal guidance and control and to other advanced methods. A version of MAVERIC was delivered that flies these alternate vehicles in 3DOF.

There are many items to be examined in determining success of the simulations in actually being able to fly a vehicle. For the X-33, there are a number of constraints that must be satisfied during flight. Transport delays will impact the ability of the control systems to control the vehicle. Constraints and other success criteria include

- Overall structural constraints such as acceleration and dynamic pressure times angle of attack

- Component structural constraints such as hinge moments and root loads

- Body rate limits

- Durations at minimum and maximum aerosurface positions

- Aerothermal indicator limits and targets

- Reaction Control System propellant, usage and firing behavior versus constraints

- Behavior of the flight profile (oscillations, chattering, etc.)

- Main engine cutoff conditions (altitude, Mach)

- Terminal Area Energy Management interface conditions (altitude, downrange, Mach, heading, high/low energy) 
- Touchdown conditions (does the flight reach the runway?)

The following emphasis will be placed on test successes and failures.

\section{Most Important}

Design cycle time for vehicle configuration update

Effort required for new missions

Success of flight (or percentage of dispersions)

Significant constraint violations

Performance consistency between missions

Lack of large transients (at maneuvers, at PPO, etc.)

Applicability to other vehicles

\section{More Important}

Number of mission dependent data parameters

Size of mission dependent data parameters

Verification difficulty

Algorithm complexity

Design process automation capability

Ease of modification

Control duty cycles

Acceptable attitude profiles

The following parameters will be checked against constraints for each test:

Aerosurface deflections

Flaps

Elevons

Rudders

Hinge moments

Flaps

Elevons

Rudders

Attitude Errors

RCS propellant

Number of simultaneous RCS thrusters firing

PLAD pressure

PLAD mass

Body rates

Roll, pitch, yaw

Alpha, beta, bank

\section{Less Important}

Number of mission independent data parameters

Size of mission independent data parameters

Execution time

Algorithm modularity (or can it be made modular) 
Down-selection to alternate flight mode (trajectory reshape, alternate landing site, etc.)

The following parameters will be checked against constraints for each test: Load indicators

Q-alpha

Q-beta

Shear vs. Bending

Canted fin

Vertical Fin

Axial acceleration

Normal acceleration

Maximum dynamic pressure

Thermal indicators

\section{Least Important}

Number of lines of code

Pre-flight verification complexity

\section{Test Requirements}

The designs will be tested against all requirements that apply. The test list indicates which are for guidance, control, or both. The tests are comprised primarily of simulations and computing system requirements. Control system designers will be required to show that their design meets all frequency domain requirements.

Guidance simulations will be mostly 3DOF. Controls engineers will inspect 3DOF results to ensure that the vehicle will be able to fly what the guidance scheme is commanding. A few guidance cases will be run in 6DOF, although the nominal control system will not be re-tuned to the new guidance. The tests will be run for insight as to the controllability of the guidance commands. Control system simulations will be 6DOF. If the given guidance or control design does not apply to all phases of flight, it will be tested for those phases for which it applies.

All simulation tests will be performed on the edalf1 computer at Marshall Space Flight Center. Overall requirements for each simulation test are that vehicle control is maintained throughout, that it is successfully delivered to its destination within the required accuracy, and that no vehicle constraints are violated.

Constraints are primarily load and thermal indicators and those values will be provided for development testing. There should be no large transients.

All control methods will be tested with the nominal $X-33$ guidance system. $A$ modified guidance will be used for RTLS simulations. 
Tests are focused on rocket-powered launch vehicles rather than airbreathing/rocket combined cycle propulsion vehicles. These will not be tested in simulation due to their radical differences in flight dynamics properties as compared to the X-33. In particular, flight at very high dynamic pressure for sustained periods, with engine performance dependent upon angle of attack, is required. Developers of advanced guidance and control schemes should describe how their methods would be applied to these vehicle types, if the methods are applicable for airbreathing vehicles.

\section{A. Nominal Flight Tests}

The multiple vehicle types and missions below are intended to show the flexibility of the candidate methods to fly different vehicles and missions. Differing missions should be flown without any modification except for I-loads that do not require pre-flight analysis effort. Different vehicles may be flown with modifications, but these should be minor.

Different vehicle and mission simulations have been developed by MSFC to emulate the particular flight dynamics desired. The different vehicles require modifications to the $X-33$ vehicle simulation (e.g. increased specific impulse of the main engines), but the intent is to minimize the change in the physical characteristics of the $X-33$ vehicle (i.e. minimize the perturbations to the plant model). Different vehicles are indicated below by a vehicle number in brackets. The full set of alternate vehicles will be used for guidance algorithms. Control algorithms will fly the RTLS trajectories only, as the control algorithms should be sufficiently tested otherwise by the full set of X-33 tests. Reference trajectories and/or MAVERIC simulations were made available for the alternate vehicles.

It should be noted that a criteria for goodness of the algorithm is how much analysis work is required to adapt to new cases, with less being better. For example, the same vehicle going to different orbits, coming back from different orbits, or landing at different landing sites should not require significant work for each case. It will be considered to be the same vehicle flying a different mission.

\section{$\underline{\text { Nominal Mission }}$}

- X-33 10a1 nominal trajectory. Dispersion tests included.

- X-33 10d1 nominal trajectory. Dispersion tests included.

- Modified [1] (artificially high Isp) X-33 flight to orbit from KSC to $28.5 \mathrm{deg}$ LEO. Dispersion tests included. This case tests guidance accuracy and performance for orbital delivery.

- Reentry [2] from 28.5-deg LEO to nominal landing site. X-33 vehicle characteristics, not very constrained by thermal needs. Dispersion tests included. This case tests guidance accuracy and performance for entry.

- Same [2] as above but very constrained by thermal indicators. It should be noted that the entry guidance will be evaluated partly on its ability to maintain 
thermal indicators within limits in the presence of dispersions. Dispersed tests included. This case tests the ability of the guidance to target TAEM conditions while minimizing thermal dispersions.

- Reentry [3] with reduced lift/drag ratio as compared to X-33. Dispersion tests included. This case tests the adaptability of the guidance to lower L/D vehicles.

- Modified [1] X-33 flight to orbit from KSC to 51.6 deg ISS rendezvous phasing orbit (artificially high Isp). This cases tests the ability of the guidance to target different orbits without modification.

- Reentry [2] from ISS orbit to nominal landing site. Low crossrange. This case tests the ability of the guidance to return from orbit without modification. Fly both with the entry profile from the fourth case above and one tailored to this case.

- Same [2] as the fourth case above but large crossrange. Dispersion cases included. This case tests the capability of guidance to target large crossrange cases without modification.

The above tests will be run primarily in 3DOF for guidance and 6DOF for control designs.

\section{B. Aborts}

- X-33 10a1 PowerPack-out aborts at different trajectory times. This will include cases that fly to an alternate landing site. One of the early cases will include a trajectory that is very constrained by vehicle control capabilities. Dispersions for some of these aborts.

- Modified [1] X-33 RTLS abort for orbital insertion missions; engine out at 3 different times (shortly after liftoff, near maximum dynamic pressure, and near the latest possible time). Dispersion tests for one of these. The latest time will emulate the fly-back portion of a fly-back booster. This tests multiple flight conditions that would not be tested otherwise.

- Same as the latest abort from the bullet above but with a much lower LD; return more ballistic [3]. Last RTLS time only. Models the fly-back portion of a fly-back booster.

- Reentry [2] to abort landing sites from 28.5-deg LEO orbit. Tests the ability of the guidance to target a different landing site without modification.

- Failures of various vehicle subsystems. Failures may include complete or partial failure of aerodynamic control surfaces and single or multiple reaction control thruster failures (tests for control designs only). Failure of one elevon (half of one side). Partial TVC failure through valve failure.

- Cases where the vehicle is faced with unforeseen problems. Situations like that which occurred on the first Delta III, where a rolling mode existed that was not expected to be important. Situations like that which occurred on the first Pegasus XL, where the aerodynamic data was not correct. Situations like that which occurred on a recent Japanese M5 flight, where the first stage 
performed miserably and the second stage could not compensate sufficiently to reach orbit.

For aborts and for cases that are identified where the vehicle can recover from failure, some of these may be run with Monte Carlo dispersions.

Abort cases will be run primarily in 3DOF for guidance and 6DOF for control.

\section{Dispersions}

Dispersions for nominal and abort cases will be run using the Monte Carlo technique. Algorithms will be compared to determine which are more robust to dispersions. Where appropriate, a dispersion box (variation about target final conditions) will be defined and the success of the algorithm at meeting the targets will be evaluated. Success for entry includes successful targeting of the TAEM box. The success of the algorithms in keeping the vehicle within constraint boundaries (thermal, loads, controls) will also be evaluated.

Some individual stressing cases will be developed for test. A few will be intentionally far off-nominal in order to see how the algorithms adapt.

The dispersion space that must be accommodated includes but is not limited to aerodynamics, engine performance, winds and gusts, mass properties, actuator bandwidth, flex mode frequency and damping, and slosh mass, location, frequency, and damping.

The dispersion tests will be run in 3DOF for guidance and 6DOF for control.

\section{Frequency Domain Tests}

Verification of the control design in the frequency domain is accomplished in offline tools developed by the investigators. Frequency response analysis should include sufficient detail to allow comparison to alternate designs and could include the basic airframe transfer functions, open-loop stability margins and linear time response to step inputs. The frequency domain analyses of the flight control system must verify open-loop stability at various operating points for the different phases and the different trajectories/vehicles.

\section{E. Ease of Modification}

What is the difficulty for a practicing engineer to modify the design to handle a new mission or vehicle or abort case? The developer will document the process used to transfer between different vehicles, missions, and aborts. A MSFC engineer will apply this process to a similar class vehicle. The objective will be to demonstrate that the documentation is sufficiently detailed for use and that the process for implementing the design on a different vehicle is not too difficult. 


\section{F. Trajectory Design}

Any closed-loop guidance that automatically modifies trajectories must include path constraints in its design. These are typically thermal, control, and loads constraints. Loads constraints include maximum axial and normal acceleration, maximum dynamic pressure, and maximum Q-alpha or Q-beta. A typical thermal constraint is the peak heat rate. Control constraints take the form of angle of attack (and sideslip) and Q-alpha constraints for nominal and engine out cases. There are cases where the vehicle needs to fly high angles of attack to recover from an engine failure, but does not have the control power to fly certain desired values of alpha (some early X-33 powerpack-out aborts fall in this category).

\section{G. Computing Requirements}

Computer limitations are important for algorithms that might fly a reusable launch vehicle any time soon. However, algorithms that are otherwise good but do not satisfy the constraints here will not be discarded just because they are more computer-intensive.

CPU: The current X-33 flight Vehicle Management Computers are approximately 60 MIPS machines. This is approximately $1 / 42$ of the speed of one CPU on the edalf 1 computer. If we assume the Flight Manager computer on X-33 uses $1 / 3$ of its time for guidance, $1 / 3$ for control, and $1 / 3$ for other functions (such as navigation processing, Propellant Utilization system, etc.), this gives us a requirement for speed to fit on the $X-33$ computers.

The guidance and control are required to fit within these constraints if they are to be satisfactory for today's flight computers. If a guidance or control design does not satisfy this requirement, but otherwise meets all robustness requirements, it will be judged to be useful for future reusable launch vehicles as computing capabilities catch up. To be available for flight test immediately subsequent to the completion of the evaluation effort associated with this project, the design must meet this CPU requirement.

As an example, if a guidance scheme operates at $1 \mathrm{~Hz}$, it would use no more than (1/42) times (1/3) seconds, or no more than $1 / 126$ second on the edalf1 for one computation cycle. A control scheme that operates at $50 \mathrm{~Hz}$ would use no more than $(1 / 50)$ times $(1 / 42)$ times $(1 / 3)$ seconds, or no more than $1 / 6300$ second on the edalf 1 for one computation cycle. The CPU time can be measured using an internal CPU clock function.

Memory: The total memory allocated to the GN\&C subsystem on the X-33 flight computer should not be exceeded by the proposed guidance or control design, with allowances for the other software in the computer. If a guidance or control design does not satisfy this requirement, but otherwise meets all robustness 
requirements, it will be judged to be useful for future reusable launch vehicles as computing capabilities catch up.

\section{Summary}

The Advanced Guidance and Control Project is a $11 / 2$-year effort to find methods that will reduce the cost and time needed for reusable launch vehicle design and flight operations, and will significantly increase safety and reliability. Six independent control schemes, three ascent/abort guidance methods, and three entry guidance designs are being developed and tested. The tests will demonstrate which of these methods are robust to vehicle, mission, and environmental changes, and to aborts, failures, and different vehicle designs. A fly-off will be conducted to find the methods that yield the most benefits. These methods will be available for all potential builders of future reusable launch vehicles.

\section{References}

1. Charles E. Hall, Michael W. Gallaher, and Neal D. Hendrix, "X-33 Attitude Control System Design for Ascent, Transition, and Entry Flight Regimes," paper AIAA 98-4411, AIAA Guidance, Navigation, and Control Conference, Boston, MA, Aug. 1998.

2. John M. Hanson, Dan J. Coughlin, Gregory A. Dukeman, John A. Mulqueen, and James W. McCarter, "Ascent, Transition, Entry, and Abort Guidance Algorithm Design for the X-33 Vehicle," paper AIAA 98-4409, AIAA Guidance, Navigation, and Control Conference, Boston, MA, Aug. 1998.

3. Dan Moerder, "Neighboring Optimal Control Approach to Ascent Guidance," 2000 AIAA Guidance, Navigation, and Control Conference, Denver, CO, Aug. 2000.

4. Ping Lu, "Nonlinear Trajectory Tracking Guidance with Applications to a Launch Vehicle," Journal of Guidance, Control, and Dynamics, Vol. 19, No. 1, Jan-Feb 1996.

5. Philip Hattis and Richard Bailey, "Overview of the Kistler K1 Guidance and Control System," AIAA paper 99-4208, AIAA Guidance, Navigation, and Control Conference, Portland, OR, August 1999.

6. Kenneth Spratlin, "An Adaptive Numeric Predictor-Corrector Guidance Algorithm for Atmospheric Entry Vehicles," M.S. Thesis, MIT, May 1987, NASA-CR-171989. 
7. Robert D. Braun and Richard W. Powell, "A Predictor-Corrector Guidance Algorithm for Use in High-Energy Aerobraking System Studies," AIAA paper

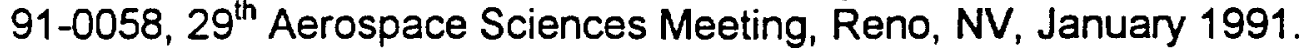

8. A. Miele and T. Wang, "Robust Predictor-Corrector Guidance for Aeroassisted Orbital Transfer," IAF paper 95-A504, International Astronautical Congress, Oslo, Norway, October 1995.

9. Richard W. Powell, "Numerical Roll Reversal Predictor-Corrector Aerocapture and Precision Landing Guidance Algorithms for the Mars Surveyor Program 2001 Missions," AIAA paper 98-4574, AlAA Atmospheric Mechanics Conference, Boston, MA, August 1998.

10. Ping Lu, "Entry Guidance by Trajectory Regulation," 2000 AIAA Guidance, Navigation, and Control Conference, Denver, CO, Aug. 2000.

11.D.T. Chen and K.D. Mease, "A 3D Predictive Entry Guidance Approach," 2000 AIAA Guidance, Navigation, and Control Conference, Denver, CO, Aug. 2000.

12. Yuri Shtessel and Charles Hall, "Reusable Launch Vehicle Control in Multiple Time Scale Sliding Modes," 2000 AIAA Guidance, Navigation, and Control Conference, Denver, CO, Aug. 2000.

13. A. J. Calise, E. Johnson, and H. El-Shirbiny, "An Adaptive Flight Control System Design for the X-33 Vehicle," 2000 AIAA Guidance, Navigation, and Control Conference, Denver, CO, Aug. 2000.

14. David B. Doman, David Leggett, and Mark Mears, "Development of a Hybrid Direct-Indirect Adaptive Control System for the X-33," 2000 AIAA Guidance, Navigation, and Control Conference, Denver, CO, Aug. 2000.

15. A. Scottedward Hodel, "Convex Programming in the Design of a Control System for a Reusable Launch Vehicle," 2000 AIAA Guidance, Navigation, and Control Conference, Denver, CO, Aug. 2000.

16. R. Smith, A. Ahmed, F.Y. Hadaegh, "The Design of a Robust Parametrically Varying Attitude Controller for the X-33 Vehicle," 2000 AIAA Guidance, Navigation, and Control Conference, Denver, CO, Aug. 2000.

17. J. Jim Zhu, Brad D. Banker, Charles E. Hall, "X-33 Ascent Flight Controller Design by Trajectory Linearization- A Singular Perturbational Approach," 2000 AIAA Guidance, Navigation, and Control Conference, Denver, CO, Aug. 2000. 